\title{
OPEN Affordable automated phenotypic antibiotic susceptibility testing method based on a contactless conductometric sensor
}

\begin{abstract}
Xuzhi Zhang ${ }^{1,2}$, Xiaoyu Jiang ${ }^{1}$, Qianqian Yang ${ }^{1}$, Yong X $\mathrm{Uu}^{1}$, Xiaochun Wang $^{1}$, Jinping Wang ${ }^{3 凶}$, Xiaobo Sun ${ }^{3}$, Guosi Xie ${ }^{1}$, Yan Zhang ${ }^{1}$, Jun Zhao ${ }^{1}$ \& Keming Qu ${ }^{1,2}$

User-friendly phenotypic antibiotic susceptibility testing (AST) methods are urgently needed in many fields including clinical medicine, epidemiological studies and drug research. Herein, we report a convenient and cost-effective phenotypic AST method based on online monitoring bacterial growth with a developed 8-channel contactless conductometric sensor (CCS). Using $E$. coli and $V$. parahaemolyticus as microorganism models, as well as enoxacin, florfenicol, ampicillin, kanamycin and sulfadiazine as antibiotic probes. The minimum inhibitory concentration (MIC) determination was validated in comparison with standard broth microdilution (BMD) assay. The total essential agreements between the CCS AST assays and the reference BMD AST assays are 68.8-92.3\%. The CCS has an approximate price of $\$ 9,000$ (USD). Requiring neither chemical nor biotic auxiliary materials for the assay makes the cost of each sample $<\$ 1$. The MICs obtained with the automated CCS AST assays are more precise than those obtained with the manual BMD. Moreover, in 72 percent of the counterpart, the MICs obtained with the CCS AST assays are higher than that obtained with the BMD AST assays. The proposed CCS AST method has advantages in affordability, accuracy, sensitivity and user-friendliness.
\end{abstract}

Determining the susceptibility of microorganisms towards target agents (e.g., antibiotics and nanoparticles) is of great significance in many fields including medicine, epidemiology, drug research, environmental surveys and agriculture ${ }^{1-3}$. Antimicrobial susceptibility testing (AST) is the concentration of antimicrobial agents that is required to inhibit proliferation of target microorganisms in vitro and is the most valuable tool for determination ${ }^{1,4}$. To achieve rapid, reproducible and accurate measurements, automated AST methods have been developed in recent decades, and most available methods are divided into two categories based on mechanism: genotypic and phenotypic methods.

Genotypic AST methods attempt to determine specific resistance genes or genetic mutations using molecular amplification $^{4}$ or sequencing ${ }^{5}$ approaches. In most of these performances, the requirements of microbial growth are bypassed resulting in a fast response; the results can be obtained with high specificity and sensitivity within 2 $\mathrm{h}^{4,6}$. An intrinsic limitation of all genotypic methods is that they detect only the potential for resistance. Thus, the susceptibility has to be validated with phenotypic tests ${ }^{1,7}$. Moreover, the other drawback with genotypic methods is that only known sequences associated with resistance can be targeted. Not only are there many more sequences that have yet to be elucidated, but new forms of resistance are out of reach ${ }^{2}$. In contrast, phenotypic AST methods analyze the effect of target agents on characteristics of microbial growth. They can provide qualitative data for the strain tested as well as quantitative values of the minimum inhibitory concentration (MIC, i.e., the lowest antibiotic concentration that inhibits visible growth $)^{4,7-10}$. To date, phenotypic AST methods are still considered the gold standard and are the most popular because of their universal detection of resistance irrespective of the resistance mechanisms ${ }^{2,8}$.

To improve the efficiency of phenotypic AST assays, various semi-automatic and automatic readout approaches have been introduced as reviewed previously ${ }^{1,4,11-13}$. In general, optics ${ }^{2,9,12-16}$, electrochemistry ${ }^{12,17-20}$,

\footnotetext{
${ }^{1}$ Yellow Sea Fisheries Research Institute, Chinese Academy of Fishery Sciences, Qingdao 266071, China. ${ }^{2}$ Laboratory for Marine Fisheries Science and Food Production Processes, Pilot National Laboratory for Marine Science and Technology (Qingdao), Qingdao 266071, China. ${ }^{3}$ College of Chemical and Pharmaceutical Sciences, Qingdao Agricultural University, Qingdao 266109, China. ${ }^{\varpi}$ email: jpwang@qau.edu.cn; qukm@ ysfri.ac.cn
} 
microcalorimetry ${ }^{21}$, mass sensor ${ }^{22}$, mass spectrometry ${ }^{23}$ and gene analysis ${ }^{8}$ techniques are used for the readout of microbial growth. These developments offer fast susceptibility results making measurements simple versus manual plate counting. Though most of these AST methods are still in the proof-of-concept status, those based on on-line monitoring microbial growth with optical devices have been implemented widely to conduct phenotypic assays $^{15,16}$, because they also offer accuracy and reliability, as well as the capacity for quantitative MIC results in a non-destructive and high-throughput manner.

Several commercial systems, including MicroScan WalkAway, Vitek, BD Phoenix and Sensititre, are already moving into clinical spaces ${ }^{13,24}$. These inventions can speed up AST, increase consistency of susceptibility results across different locations, and reduce the burden of work for users. For example, the susceptibility profile of up to 96 microbial samples can be determined using the automated broth microdilution (BMD) assay through online optical density (OD) measurements within $20 \mathrm{~h}^{25}$ despite there being no official standardized protocol for OD measurements. Automatic readout approaches can lower the accuracy depending on the skill of operators. However, the cost stunts their widespread applications, especially in the developing world ${ }^{4,5,26}$. They might also be unreliable when target microorganisms are cultured in the presence of substrates that may interfere with the optical signal or only proliferate when attached to base material surfaces ${ }^{4}$.

Electrochemical readout requires only simple electronics for direct electronic detection of microbial growth. This can bypass the requirements of complex optical-electric conversion ${ }^{18}$. Thus, instruments are easier to miniaturize and become more cost-effective versus optical systems. Traditionally, the working electrode is galvanic contact with liquid broth or solution. This invasive manner inevitably causes environmental perturbations on microbial growth as well as undesirable electrode deterioration and non-specific fouling ${ }^{27}$ that can undermine the accuracy of on-line monitoring ${ }^{12,19}$. Therefore, an electrochemical readout approach that can be used to monitor microbial growth in real time with non-invasive manner is urgently needed for developing practical AST methods.

To meet this goal, we have constructed a contactless conductometric sensor (CCS) based on a multi-channel capacitively-coupled contactless conductivity detector $\left(C^{4} D\right)^{28}$. The measurement based on this instrument offers several advantages over classical electrochemical- and turbidity/absorbance-based approaches. For instance, it is superior to optical-based methods in that turbidity and other optical interferences are significant issues ${ }^{29}$. Versus impedance sensors, this method exhibits better reproducibility and accuracy with high temporal resolution ${ }^{17,30}$. Moreover, unlike other electrochemical methods, this system requires neither chemical, biotic, or physical compounds as indicators or auxiliary materials, nor any immobilizing steps. This effectively reduces the cost and complexity for on-line monitoring of microbial growth ${ }^{12}$.

Here, we report a novel AST method by modifying a common BMD assay. An 8-channel CCS, which allows simultaneous cultivation and on-line analysis of growth inhibition, is developed and characterized. Taking $E$. coli and $V$. parahaemolyticus as microorganism model and enoxacin, florfenicol, ampicillin, kanamycin and sulfadiazine as antibiotic probes, we validated the capability of the new method for achieving MIC determination. Our goal is to overcome the limited applicability of existing methods for studying the dynamic effects of antibiotics on microbial growth kinetics, and to provide an affordable and simple tool for phenotypic AST assays.

\section{Materials and methods}

Materials and reagents. Standard bacterial strains of E. coli (ATCC35150) and V. parahaemolyticus (ATCC17802) were purchased from BIOBW Biotechnology Co., Ltd (Beijing, China). E. coli and V. parahaemolyticus isolates were obtained from fishery water and the shrimp Penaeus vannamei, respectively. Enoxacin, florfenicol, ampicillin, kanamycin and sulfadiazine were purchased from Sigma-Aldrich (St. Louis, MO, USA). Other common chemicals were purchased from the Shanghai Chemical Reagent Co. (Shanghai, China) and were of analytical grade. Unless otherwise indicated, liquid broths and solutions were prepared with ultrapure water (resistivity: $18.2 \mathrm{M} \Omega \mathrm{cm}$ at $25^{\circ} \mathrm{C}$ ) from a Master Touch-RUV water purification system (Hitech Instruments Co., Ltd., Shanghai, China).

Escherichia coli was aerobically cultured in liquid Luria-Bertani (LB) broth. V. parahaemolyticus was aerobically cultured in liquid $2216 \mathrm{E}$ broth (a common complex culture broth for marine bacteria, consisting of $0.5 \%$ tryptone, $0.1 \%$ yeast extract, $3.4 \% \mathrm{NaCl}$ and $0.01 \% \mathrm{FePO}_{4}, \mathrm{pH} 7.6-7.8$ ). Both of the broths were purchased from the Hope Bio-Technology Co., Ltd (Qingdao, China). Bacterial cultivation was conducted in accordance with previously described methods ${ }^{28,31}$ with minor modifications. Briefly, strains were stored at $-80{ }^{\circ} \mathrm{C}$ and then pre-grown overnight in the appropriate broth with constant shaking. Unless otherwise indicated, the incubation temperature for E. coli and V. parahaemolyticus was $37^{\circ} \mathrm{C}$ and $28^{\circ} \mathrm{C}$, respectively. Active strains were then further transferred to new culture broth. After a second incubation for $\sim 10 \mathrm{~h}$, the cell numbers were measured with an OD method according to Clinical and Laboratory Standards Institute (CLSI) guidelines ${ }^{32}$. The results were then validated with a plate-counting method used previously ${ }^{28}$. The cultures were immediately diluted to achieve a cell concentration of $10^{9} \mathrm{CFU} / \mathrm{mL}$ for further use.

Fabrication and characterization of the CCS. The 8-channel CCS was developed on the basis of one prototype we constructed previously ${ }^{28}$. In brief, a miniature electronic fan (MA1062, Sunon Technology Development Co., Ltd, Beijing, China), a programmable temperature sensor (WH801, Wattion Electronic Control System Co., Ltd, Guangzhou, China) and a thermoelectric cooler (TEC1-12706, Changshengyongxing Co., Ltd, Shenzhen, China) were used to guarantee expected identical temperature inside the working chamber of the sensor. A developed eight-channel $C^{4} D$ (manufactured by eDAQ Pty Ltd., Sydney, Australia), including the software TERA TERM, was used to monitor the conductivity changes of the liquid broth on-line in test tubes. Note, in order to obtain stable and sensitive conductivity change values for the liquid broth over the range of $15-45 \mathrm{mS} / \mathrm{cm}$, the geometric parameters of the working electrodes were developed according to Equations 1-5 
from the literature ${ }^{33}$. Here, in each channel a couple of copper cylinders (ID $=5.01 \mathrm{~mm}$; thickness $=0.5 \mathrm{~mm}$; length $=2.0 \mathrm{~mm}$ ) were used as the actuator electrode and pick-up electrode. The distance between the actuator electrode and pick-up electrode was $8.0 \mathrm{~mm}$. The excitation frequency and excitation amplitude were $500 \mathrm{~K} \mathrm{~Hz}$ and $16 \mathrm{~V}$, respectively. The collection period of apparent conductivity value can be selected over a range of $1 / 100 \mathrm{~s}$ to $20 \mathrm{~min}^{28,34}$. The characteristics of the electronic sensor, including the uniformity of temperature in the working chamber and the robustness and reproducibility of the $C^{4} \mathrm{D}$ system, were characterized with the same methods as used previously ${ }^{28}$ (see Supplementary Information).

Characterizing growth behaviors of $\boldsymbol{E}$. coli and $\boldsymbol{V}$. parahaemolyticus with the CCS. To characterize the growth behavior of viable E. coli, bacterial cells at desired concentrations in liquid LB broth $(2 \mathrm{~mL}$ in total, containing $0.5 \% \mathrm{NaCl}$ ) were loaded into test tubes (NORELL tubes, $\mathrm{OD}=5.0 \mathrm{~mm}$, ID $=4.2 \mathrm{~mm}$, length $=203.0 \mathrm{~mm}$, volume $\approx 2.8 \mathrm{~mL}$, Norell, Inc., Morganton, USA). The tube openings were then covered with gas-permeable films. Meanwhile, a control sample without inoculation was prepared similarly and loaded into another test tube. Each tube was then inserted into a separate channel of the CCS in which the temperature was $37^{\circ} \mathrm{C}$. After an incubation of $120 \mathrm{~s}$ (to balance the temperature inside and outside of the tubes) ${ }^{28}$, apparent conductivity values were collected every $30 \mathrm{~s}$. The apparent conductivity data from each tube were blanked by subtracting the first recordings from the remaining values to form normalized apparent conductivity values (NACVs, showed in voltage). The CCS growth curves were then generated by plotting NACVs as a function of incubation times. In the case of characterizing growth behavior of viable $V$. parahaemolyticus at $28{ }^{\circ} \mathrm{C}$, liquid $2216 \mathrm{E}$ broth was used instead of LB broth.

CCS AST assay. Scheme 1 shows that the procedure of the phenotypic AST assay with the CCS consisted of two steps: Preparation of AST samples and incubation/read out. Re-suspended bacterial cells in liquid broth were added to each tube for a final concentration of approximately $5 \times 10^{5} \mathrm{CFU} / \mathrm{mL}^{23}$. In the test tubes, serial twofold dilutions of antibiotics were made in liquid broth. After covering the tube opening with a gas-permeable film, we simultaneously inserted all tubes into a separate channel of the CCS and collected the NACVs as stated in "Characterizing growth behaviors of E. coli and V. parahaemolyticus with the CCS" for $20 \mathrm{~h}$. The liquid broth with and without bacterial cells were taken as positive and negative controls, respectively. In total, 2400 datapoints were obtained for every tested sample. Bacterial growth curves were simultaneously generated by plotting NACVs against incubation times. The MIC for each antibiotic is defined as the lowest antibiotic concentration, which inhibits the growth of the target microorganism, as assessed from the absence of sigmoidal curve ${ }^{9}$.

BMD AST assay. In the reference experiments, the MICs of these five kinds of antibiotics against E. coli and V. parahaemolyticus were determined by the BMD method according to the CLSI guidelines ${ }^{32}$. Briefly, each antibiotic was measured in two-fold concentrations over a desired range in Corning 96-well plates (Corning Incorporated, USA). The final bacterial inoculum in the measurement was approximately $5 \times 10^{5} \mathrm{CFU} / \mathrm{mL}$. In each well, $100 \mu \mathrm{L} \mathrm{LB}$ or $2216 \mathrm{E}$ was used as nutrient broth for E. coli and V. parahaemolyticus, respectively. Plates were incubated for $20 \mathrm{~h}$. The MIC was defined as the lowest concentration of antibiotic, resulting in complete inhibition of growth as determined visually ${ }^{23}$.

Precision and validity analysis. A precision analysis was conducted referring to Kenneth and Kirby's report $^{35}$ with minor modifications. Briefly, CCS AST and BMD AST assays were repeated 11 times in triplicate for each antibiotic-microorganism combination using kanamycin as an antibiotic probe. All performances occurred on separate days with freshly prepared antibiotic dilutions and independent inocula were used to determine the MICs. The distributions of determined MICs from the two assays were analyzed in comparison. Validity analysis was conducted with essential agreement (EA) levels using 65 E. coli isolates and $16 \mathrm{~V}$. parahaemolyticus isolates against enoxacin, florfenicol, ampicillin, kanamycin and sulfadiazine. Each MIC value determined by the CCS AST assay was compared with the counterpart determined by the BMD AST assay. The CCS AST assay was considered to have an evaluable EA if its MIC was within $\pm 200 \%$ of that obtained with the reference assay. In addition, when the proportion of MIC obtained with the CCS AST assay to that obtained with the reference assay was $> \pm 2$ but $\leq \pm 3$, it was calculated as the minor error $(\mathrm{mE})$.

\section{Results}

Characteristics of the CCS. Bacterial growth is temperature-sensitive as is the response of $\mathrm{C}^{4} \mathrm{D}^{36}$. Thus, the temperature control features were characterized first. Figure S1 (Supporting information) shows the curves of 50.0 and $120.0 \mathrm{mM} \mathrm{KCl}$ solutions simultaneously measured in separate channels. As expected, the variation in apparent conductivity was very small $(<0.8 \%)$ over a period of $20 \mathrm{~h}$ in both cases. These results demonstrate the uniformity of temperature in the working chamber as well as robust and identical conductometric measurements. The difference of apparent conductivity between separate channels from the same concentration of $\mathrm{KCl}$ solution resulted from minor variations in the geometry size of the tubes and their coupling to the electrodes ${ }^{28}$. For monitoring bacterial growth, the critical factor of measurement was to record conductivity changes rather than absolute conductivity values. Thus, these differences between separate channels have no influence on forming bacterial growth curves because of our normalized algorithm. There is a linear relationship between the concentration of $\mathrm{KCl}$ solutions and apparent conductivity values with a slope of $562 \pm 3 \mathrm{mV}$ per $\mathrm{mS} / \mathrm{cm}\left(R^{2} \geq 0.9910\right)$ from 20.0 to $200.0 \mathrm{mM}$, thus suggesting a high sensitivity. 


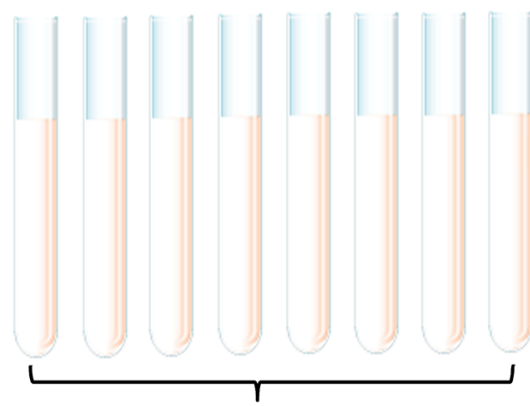

Broths loaded in test tubes

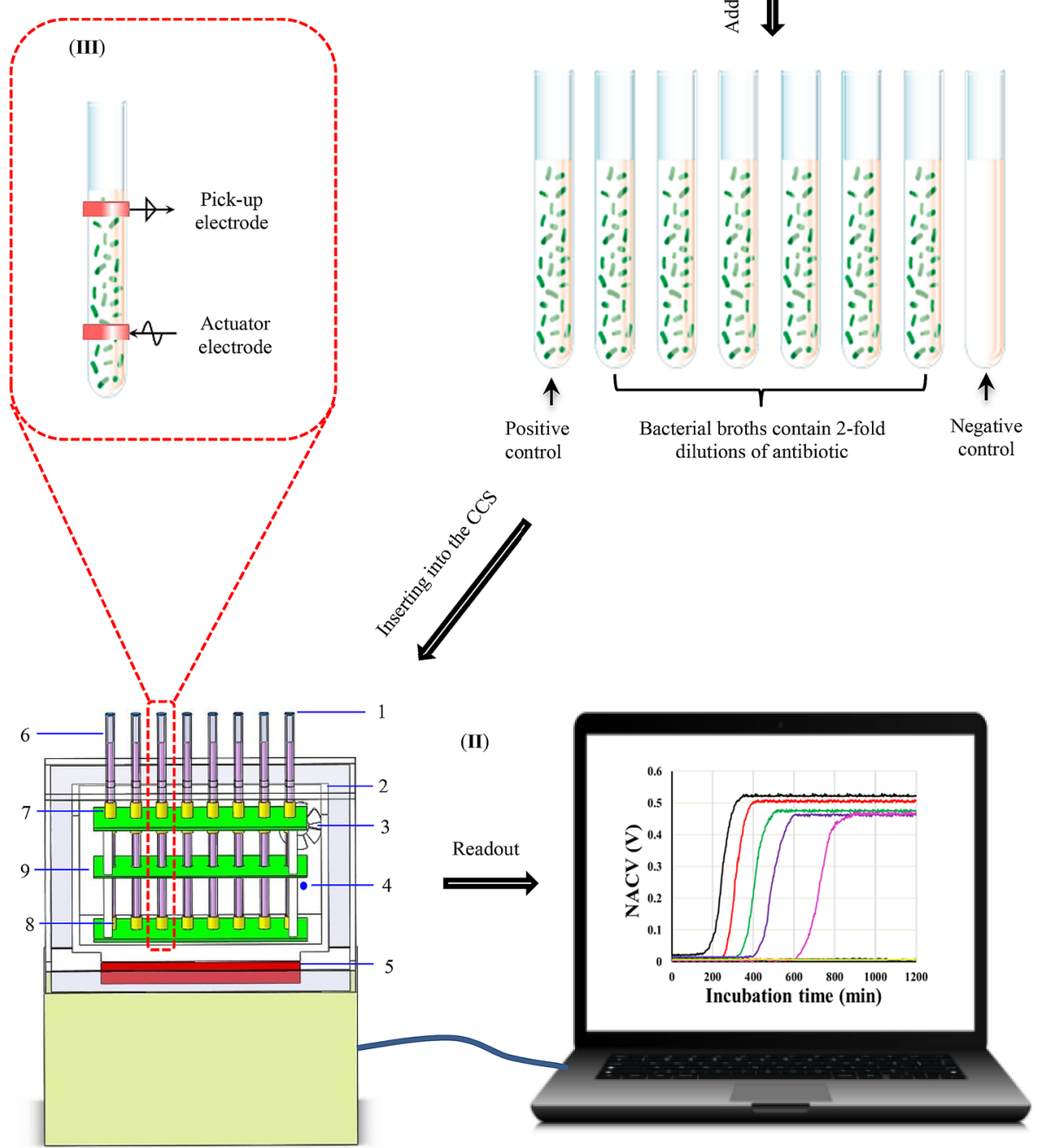

Scheme 1. Schematic diagram of the workflow of phenotypic AST assay with the 8-channel CCS. (I) Preparation of AST samples; (II) Incubation and automated generation of growth curves with the CCS controlled by a computer. 1-gas-permeable film; 2-thermal insulator; 3-mini electronic fan; 4-temperature sensor; 5-thermoelectric cooler; 6-test tube; 7-actuator electrode; 8-pick-up electrode; and 9-grounded Faraday shield to avoid direct capacitive coupling between the actuator electrode and pick-up electrode ${ }^{33}$. (III) The illustration of the couple of working electrodes outside the test tube. 

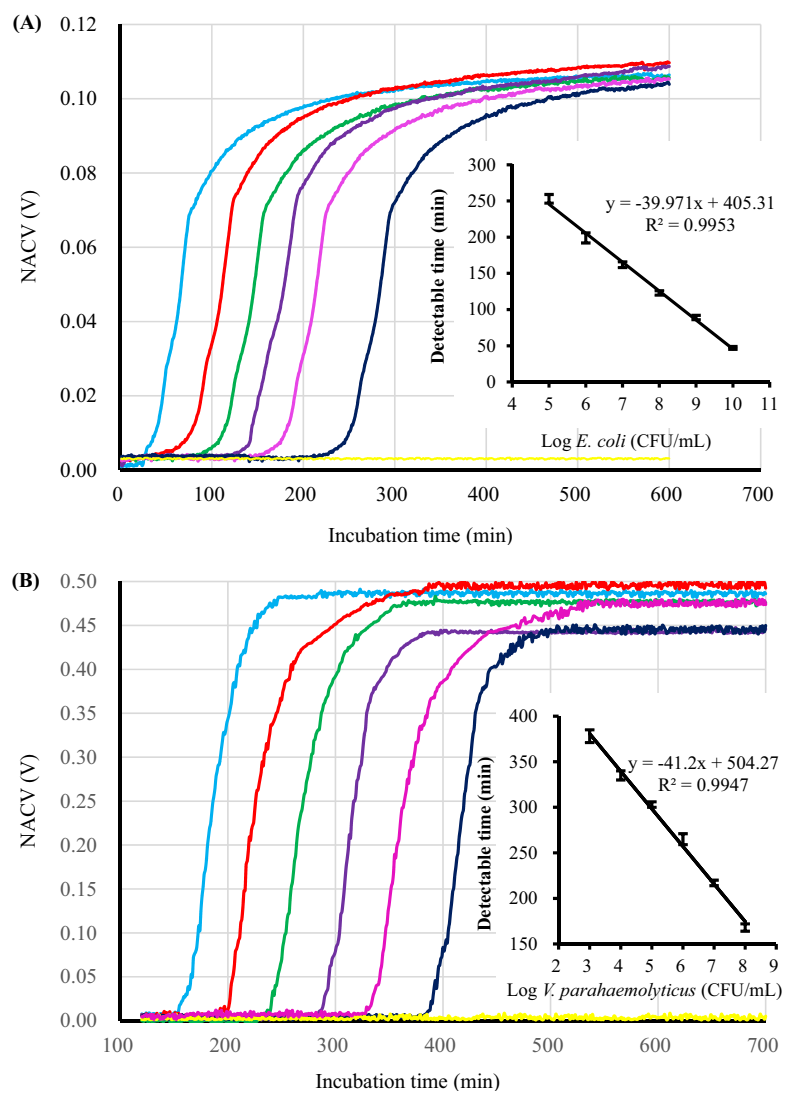

Figure 1. CCS growth curves (NACV vs. incubation time) of E. coli in liquid LB broth (A) and $V$. parahaemolyticus in liquid $2216 \mathrm{E}$ broth (B). (A): from left to right, the initial inoculum of E. coli was $10^{10}, 10^{9}$, $10^{8}, 10^{7}, 10^{6}$ and $10^{5} \mathrm{CFU}$, respectively. Insert: The linear relationship between the logarithm of initial E. coli and detectable time. (B): from left to right, the initial inoculum of $V$. parahaemolyticus was $10^{8}, 10^{7}, 10^{6}, 10^{5}, 10^{4}$ and $10^{3} \mathrm{CFU}$, respectively. Insert: The linear relationship between the logarithm of initial V. parahaemolyticus and detectable time. Yellow horizontal lines show the results of negative control experiments. NACV values of liquid broths in each test tube were collected at an interval of $30 \mathrm{~s}$ with excitation frequency of $500 \mathrm{~K} \mathrm{~Hz}$ and excitation amplitude of $16 \mathrm{~V}$.

Growth behaviors of $E$. coli and V. parahaemolyticus. The conductivities of culture broths in six test tubes, in which the initial concentration of $E$. coli cells were $10^{3}, 10^{4}, 10^{5}, 10^{6}, 10^{7}$ and $10^{8} \mathrm{CFU} / \mathrm{mL}$, respectively, were monitored simultaneously with the CCS. As shown in Fig. 1A, six sigmoidal CCS growth curves presents, meaning that the system can characterize the growth rate of viable bacteria as well as estimate the initial concentration of the initial bacterial suspension ${ }^{28,37}$. For simplicity, the time needed for the NACV of the broth to reach $0.02 \mathrm{~V}$ is defined as a "detectable time." There is a linear relationship between the logarithmic values of initial inoculum of $E$. coli and detectable times over the tested range with a correlation coefficient $\left(R^{2}\right)$ of 0.9953 (Fig. 1A insert). For the negative control (in yellow), no sigmoidal growth curve was observed over the same time span, suggesting that without viable bacteria there is no conductivity change during the incubation. These outcomes are in excellent agreement with those observed with an earlier generation prototype ${ }^{28}$.

Using the previous prototype ${ }^{28}$, we can obtain stable and sensitive conductivity change values for the liquid broth over the range of $9-14 \mathrm{mS} / \mathrm{cm}$. With the latest version, we can obtain stable and sensitive conductivity change values for the liquid broth over the range of $15-45 \mathrm{mS} / \mathrm{cm}$. This wider working window makes it possible to monitor bacterial growth in common liquid broths, e.g., LB and $2216 \mathrm{E}$.

The conductivities of culture broths in five test tubes, in which the initial concentration of $E$. coli cells were all $10^{3} \mathrm{CFU} / \mathrm{mL}$, were monitored simultaneously with the CCS. The standard deviation of detectable times is $3.2 \mathrm{~min}$, indicating a good accuracy.

For V. parahaemolyticus, the growth of serial inocula at concentrations of $10^{3}, 10^{4}, 10^{5}, 10^{6}, 10^{7}$ and $10^{8} \mathrm{CFU} /$ $\mathrm{mL}$ in liquid $2216 \mathrm{E}$ broth was simultaneously monitored with the CCS. As shown in Fig. 1B, six sigmoidal growth curves are observed. Similar to E. coli, the duration of the lag phase is proportional to the initial inoculum of bacteria. There is a linear relationship between the logarithmic values of initial inoculum of $V$. parahaemolyticus and detectable times over the tested range with a correlation coefficient $\left(R^{2}\right)$ of 0.9947 (Fig. 1B insert). No sigmoidal growth curve was observed during the incubation when the initial inoculum was zero (negative control). 
CCS AST assay. E.coli ATCC 35150 in liquid LB broth was cultured in the presence of enoxacin, florfenicol, ampicillin, kanamycin, or sulfadiazine. Figure 2A shows CCS growth curves. For the positive control (with bacteria but without antibiotic) samples, sigmoidal curves were obtained as expected. We note that these curves have considerably higher temporal resolution (at an interval of $0.5 \mathrm{~min}$ ) than those obtained with the OD method ${ }^{26}$ and the electrochemical method ${ }^{17}$. There is a lag phase for approximately $210 \mathrm{~min}$; this is possibly caused by the stress that bacteria might experience after dilution and/or a loading step as well as the time required for generating enough end products to produce detectable increasing conductivity ${ }^{28}$. The lag phase is followed by an acceleration phase during which the growth rate increases until a constant growth rate is achieved, i.e., entering the exponential phase. Subsequently, the growth rate begins to decline to form a deceleration phase. Compared to the positive control, the lag phase duration of bacteria regularly extends with increasing antibiotic concentration, thereby contributing to a delayed onset of growth when microorganisms are exposed to sub-lethal antibiotic concentrations. These data suggest that all five antibiotics show concentration-dependent effects on $E$. coli growth dynamics ${ }^{38}$.

Similar maximum growth rates and maximum growths were found in the presence of $15.625,31.250$ and $62.500 \mathrm{ng} / \mathrm{mL}$ enoxacin despite the onset of growth delays with the increase of antibiotic concentration (Fig. 2A(a)). In contrast, the maximum growth rate of $E$. coli is lower in the presence of $125.000 \mathrm{ng} / \mathrm{mL}$ enoxacin, though the final NACV at the measurement endpoint can reach a similar value to the positive control. In the presence of $0.250 \mu \mathrm{g} / \mathrm{mL}$ enoxacin, no sigmoidal growth curve was observed over the same incubation, similar to the response of the negative control. This complete inhibition suggests an MIC of $0.250 \mu \mathrm{g} / \mathrm{mL}$. The presence of low concentrations of florfenicol $(0.125,0.250$ and $0.500 \mu \mathrm{g} / \mathrm{mL})$ delayed the onset of E. coli growth and slightly lowered the maximum growth rate. The presence of $1.000 \mu \mathrm{g} / \mathrm{mL}$ significantly impacted the maximum growth rate as well as the maximum growth (Fig. 2A(b)). Interestingly, though the presence of 0.25 and $0.50 \mu \mathrm{g} / \mathrm{mL}$ ampicillin delayed the onset of growth, it did not impact the maximum growth rate. Meanwhile, the final NACV values are higher than that of the positive control, indicating that growth stimulation might have occurred ${ }^{39}$. However, the maximum growth rate and maximum growth were both depressed by 1.00 and $2.00 \mu \mathrm{g} / \mathrm{mL}$ ampicillin (Fig. 2A(c)).

Figure $2 \mathrm{~A}(\mathrm{~d})$ shows the response of kanamycin against $E$. coli. The maximum growth increases regularly with increases in antibiotic concentration from $0.25-1.00 \mu \mathrm{g} / \mathrm{mL}$ with an identical maximum growth rate similar to that in the positive control. Even in the presence of $2.00 \mu \mathrm{g} / \mathrm{mL}$ kanamycin, the maximum growth rate is similar to that of the positive control; the maximum growth at the endpoint of each measurement can reach a similar level for the positive control. The impact of sulfadiazine against E. coli is similar to that of florfenicol: the 8.0, 16.0 and $32.0 \mu \mathrm{g} / \mathrm{mL}$ antibiotics have little impact on the maximum growth rate and maximum growth. The presence of $64.0 \mu \mathrm{g} / \mathrm{mL}$ florfenicol depresses both the maximum growth rate and maximum growth (Fig. 2A(e)). These phenomena confirm that-in contrast to the duration of the lag phase-some antibiotics have neither a maximum specific growth rate nor a maximum growth (final cell amount). Thus, these latter two metrics are not reliable predictors for indicating a concentration-dependent inhibitory effect ${ }^{38}$. Microbial growth is completely inhibited when the concentration of the antibiotic is equal to or higher than the MIC. Thus, in the presence of high concentrations of antibiotics (value $\geq \mathrm{MIC}$ ), the responses of cultures are the same as that obtained with a negative controls ( see the NC lines in Fig. 2A).

Using the CCS, we obtained similar responses of enoxacin, florfenicol, ampicillin, kanamycin and sulfadiazine against $V$. parahaemolyticus (ATCC17802) growth in liquid 2216E broth. Lag phase durations of bacteria extend with increases in antibiotic concentration, suggesting that they all show concentration-dependent effects on growth dynamics. The sigmoidal CCS curves for the positive control samples (with bacteria but without antibiotic) have lag phases of $\sim 220 \mathrm{~min}$ (Fig. 2B). In the presence of $15.625,31.250$ and $62.500 \mathrm{ng} / \mathrm{mL}$ enoxacin, despite the onset of growth delays with an increase in enoxacin concentration, there are similar maximum growth rates and maximum growths. The maximum growth rate and maximum growth are both obviously depressed in the presence of $125.000 \mathrm{ng} / \mathrm{mL}$ antibiotic. In the presence of $0.250 \mu \mathrm{g} / \mathrm{mL}$ enoxacin, no sigmoidal growth curve is observed over the same time span like the response of the negative control (Fig. 2B(a)). The onset of $V$. parahaemolyticus growth is also delayed by florfenicol. Unlike the case of enoxacin, the increase of florfenicol concentration from 0.03125 to $0.12500 \mu \mathrm{g} / \mathrm{mL}$ regularly depresses both the maximum growth rate and the maximum growth; $0.500 \mu \mathrm{g} / \mathrm{mL}$ florfenicol can completely inhibit the growth (Fig. $2 \mathrm{~B}(\mathrm{~b})$ ). Interestingly, although the presence of $2.00 \mu \mathrm{g} / \mathrm{mL}$ ampicillin delays the onset of growth, it does not impact the maximum growth rate or maximum growth. However, the maximum growth rate and maximum growth both increasingly decreased with increasing ampicillin concentration $(4.00-16.00 \mu \mathrm{g} / \mathrm{mL})($ Fig. $2 \mathrm{~B}(\mathrm{c}))$. Figure $2 \mathrm{~B}(\mathrm{~d})$ shows the typical response of kanamycin against $V$. parahaemolyticus. Over the range of $2.00-16.00 \mu \mathrm{g} / \mathrm{mL}$, the maximum growth rate and maximum growth are only slightly impacted by the presence of kanamycin. Figure $2 \mathrm{~B}(\mathrm{e})$ shows the typical response of sulfadiazine against V. parahaemolyticus. The antibiotic at a concentration of 16.0 and $32.0 \mu \mathrm{g} / \mathrm{mL}$ invisibly impacts the maximum growth rate and maximum growth. The maximum growth rate and maximum growth are both increasingly depressed by the increase of ampicillin concentration from 64.00 to $128.00 \mu \mathrm{g} / \mathrm{mL}$.

BMD AST assay. The responses of E. coli and V. parahaemolyticus to enoxacin, florfenicol, ampicillin, kanamycin and sulfadiazine were characterized with BMD AST assays (Typical resulting pictures are shown in Figure S2). The resulting MICs are listed in Table 1 in comparison with those obtained with the CCS AST assays. The MICs of enoxacin against $E$. coli obtained with either BMD AST assay or CCS AST assay are much lower than that documented in the CLSI (Revised-2014) ${ }^{39}$. The MICs of kanamycin against E. coli obtained with BMD AST assay and CCS AST assay are also lower than that documented in the CLSI (Revised-2014) ${ }^{39}$. However, the MIC of ampicillin against V. parahaemolyticus obtained with CCS AST agrees well with that reported by Lopatek et al. ${ }^{40}$. 
(A: E.coli)
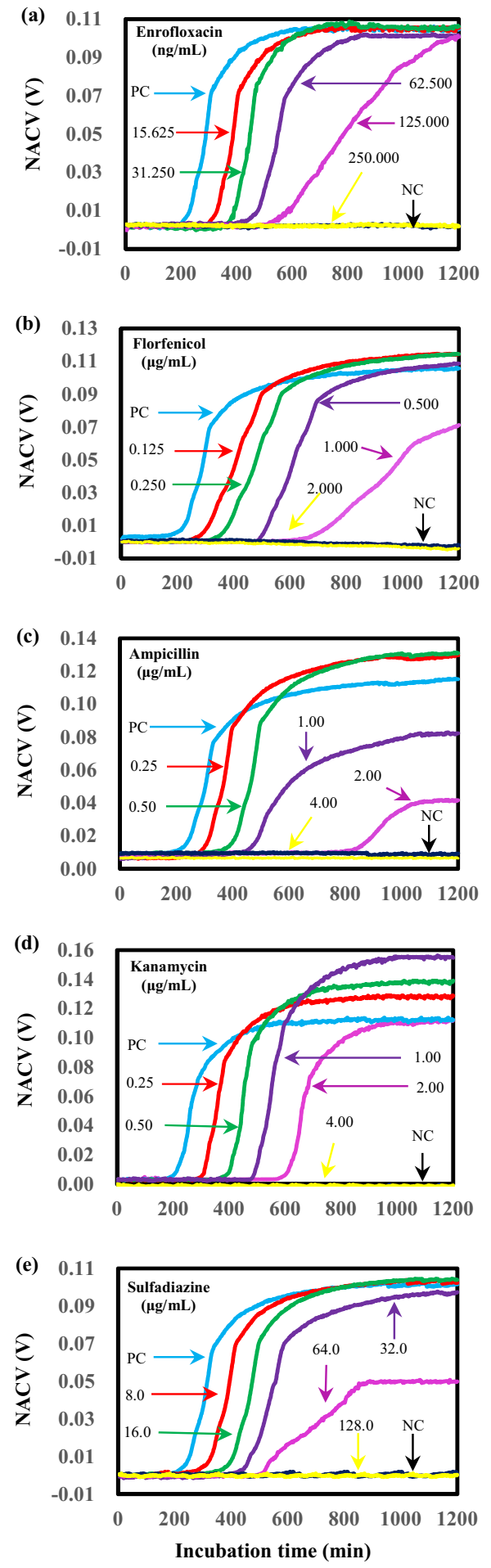

(B: V.parahaemolyticus)
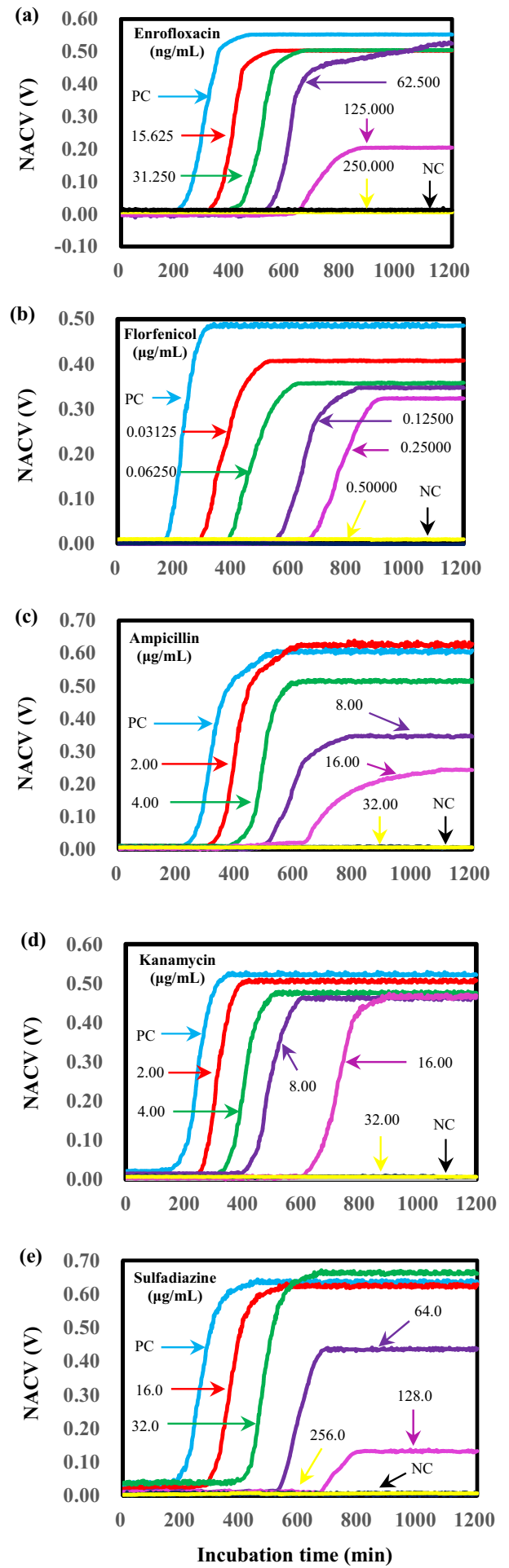

Figure 2. CCS growth curves (NACV vs. incubation time) of E. coli (A) and V. parahaemolyticus (B) in the presence of antibiotics. E. coli in liquid LB broth and $V$. parahaemolyticus in liquid $2216 \mathrm{E}$ broth were aerobically incubated at $37^{\circ} \mathrm{C}$ and $28^{\circ} \mathrm{C}$, respectively.

Precision and validity. In the precision analysis, 87.9\% (29/33) E. coli ATCC 35,150 samples show MIC of kanamycin is $4.00 \mu \mathrm{g} / \mathrm{mL}$ obtained with the CCS AST assay. In contrast, 78.8\% (26/33) E. coli ATCC 35,150 samples show a MIC of $2.00 \mu \mathrm{g} / \mathrm{mL}$ with the BMD AST assay. In the case of $V$. parahaemolyticus (ATCC17802), 


\begin{tabular}{|l|l|l|l|l|}
\hline Antibiotics & Bacterial species & CCS MICs $(\boldsymbol{\mu g} / \mathbf{m L})$ & BMD MICs $(\boldsymbol{\mu g} / \mathbf{m L})$ & Reference MICs $(\boldsymbol{\mu g} / \mathbf{m L})$ \\
\hline Enoxacin & E. coli & 0.25 & 0.25 & $2-8^{39}$ \\
\hline Florfenicol & E. coli & 2.00 & 1.00 & - \\
\hline Ampicillin & E. coli & 4.00 & 1.00 & - \\
\hline Kanamycin & E. coli & 4.00 & 2.00 & $16-64^{39}$ \\
\hline Sulfadiazine & E. coli & 128.0 & 64.0 & - \\
\hline Enoxacin & V.parahaemolyticus & 0.250 & 0.125 & - \\
\hline Florfenicol & V.parahaemolyticus & 0.500 & 0.250 & - \\
\hline Ampicillin & V.parahaemolyticus & 32.00 & 8.00 & $32^{40}$ \\
\hline Kanamycin & V.parahaemolyticus & 32.00 & 16.00 & - \\
\hline Sulfadiazine & V.parahaemolyticus & 256.0 & 128.0 & - \\
\hline
\end{tabular}

Table 1. Results of BMD AST assay in comparison with that of CCS AST assay.

\begin{tabular}{|l|l|l|l|l|l|l|}
\hline Antibiotics & Bacterial species & Total isolates & CCS MICs $(\mu \mathrm{g} / \mathrm{mL})$ & BMD MICs $(\mu \mathrm{g} / \mathbf{m L})$ & EA\% & mE\% \\
\hline Enoxacin & E. coli & 65 & $0.125-8.000$ & $0.125-4.000$ & 86.5 & 10.4 \\
\hline Florfenicol & E. coli & 65 & $1.00-64.00$ & $0.50-64.00$ & 76.9 & 23.1 \\
\hline Ampicillin & E. coli & 65 & $1.00-64.00$ & $0.500-32.00$ & 84.6 & 13.8 \\
\hline Kanamycin & E. coli & 65 & $2.00-128.00$ & $1.00-128.00$ & 92.3 & 7.7 \\
\hline Sulfadiazine & E. coli & 65 & $64.00-512.00$ & $32.00-256.00$ & 89.2 & 10.8 \\
\hline Enoxacin & V.parahaemolyticus & 16 & $0.125-16.000$ & $0.125-16.000$ & 68.8 & 31.2 \\
\hline Florfenicol & V.parahaemolyticus & 16 & $0.25-64.00$ & $0.25-64.00$ & 87.5 & 6.3 \\
\hline Ampicillin & V.parahaemolyticus & 16 & $16.00-128.00$ & $8.00-64.00$ & 81.3 & 12.5 \\
\hline Kanamycin & V.parahaemolyticus & 16 & $16.00-128.00$ & $8.00-64.00$ & 87.5 & 12.5 \\
\hline Sulfadiazine & V.parahaemolyticus & 16 & $128.0-1024.0$ & $64.0-1024.0$ & 75.0 & 25.0 \\
\hline
\end{tabular}

Table 2. Validity analysis of the CCS AST assay in comparison with the BMD AST assay.

$84.8 \%(28 / 33)$ of the samples show that the MIC of kanamycin is $32.00 \mu \mathrm{g} / \mathrm{mL}$ obtained with the CCS AST assay. The BMD assay offers $72.7 \%$ (24/33) samples with a MIC of $16.00 \mu \mathrm{g} / \mathrm{mL}$. These findings show that the CCS AST assay is superior to the BMD AST assay in terms of precision.

In total, 81 isolated bacterial strains, including $65 \mathrm{E}$. coli and $16 \mathrm{~V}$. parahaemolyticus, were used to validate the CCS AST method for the five antibiotics, also using BMD AST method as reference. The results are similar to those obtained with standard strains (Table 2). With the evaluation parameters suggested by the FDA ${ }^{9}$, we calculated the EA, as well as discrepancies defined as $\mathrm{mE}$, by comparing the MICs of the same isolate determined with CCS AST assay and that with BMD AST assay.

\section{Discussion}

MIC measurements are important in medicine, epidemiology, drug research, environmental surveys, agricultural production $^{1-3}$ and materials research ${ }^{38}$. Liquid suspension growth-based methods, such as the BMD, are gold standards for phenotypic AST assays. To monitor growth with real-time patterns, AST assays can yield results sooner than solid-phase tests ${ }^{2}$. Traditional manual BMD assays tend to be very laborious with poor precision; thus, various semi-automatic and automatic methods have been developed in the past decades ${ }^{1,4}$. These methods include on-line monitoring of microbial growth with OD patterns, which have good practicability and have been successful commercially because of their accuracy, reliability and non-destructive and high-throughput nature ${ }^{13}$. However, there are still two salient issues: (1) the optical interference from complex substrates in the broth and from adherent cells in proliferation ${ }^{37}$; and (2) the price of the instrument and cost of each sample test ${ }^{4}$. These challenges provided the motivation to develop the CCS AST method proposed herein.

The growth of bacteria transforms uncharged or weakly charged substrates, e.g., yeast, peptone and sugar into highly charged end products, such as amino acids, aldehydes, ketones, acids and other metabolites, causing a conductivity increase of the liquid broth ${ }^{41}$. This change can be monitored on-line with the sensitive CCS to generate bacterial growth curves by plotting NACVs as a function of incubation time. Using this approach, we could monitor up to eight samples for high-throughput testing.

Using standard bacterial strains of E. coli (ATCC35150) and V. parahaemolyticus (ATCC17802) as model microorganisms, the total EAs and mEs between the CCS AST assays and the reference BMD AST assays were found to be $80 \%$ and $20 \%$, respectively. For E. coli isolates, the EAs of enoxacin, florfenicol, ampicillin, kanamycin and sulfadiazine were no lower than 76.9\%. Meanwhile, the mEs were 7.7-23.1\%. For V. parahaemolyticus isolates, the EAs of these five antibiotics were no lower than $68.8 \%$ with $\mathrm{mEs}$ of $6.3-31.2 \%$. Note, these plates were incubated for the same time span as the CCS AST assays $(20 \mathrm{~h})$ to avoid differences in MIC resulting from 
different incubation times ${ }^{38}$. These outcomes thus demonstrate the accuracy of the new method, not only for standard bacterial strains but also for isolates.

The MICs obtained by the CCS AST assay are almost identical to those obtained by standard BMD assays for five different types of antibiotics against isolates. Notably, given that the readout is effectively an operatorindependent method (i.e., automated reading bypassing operator $\operatorname{error}^{8}$ ), it is not surprising that the CCS AST assay is more precise than the standard manual $\mathrm{BMD}^{35}$ (Table 2). In 72 percent of the counterpart assays, the MICs obtained with the CCS AST are higher than that obtained with the BMD AST, thus demonstrating that the automated method is more sensitive and thus more reliable. Moreover, versus endpoint measurements of BMD assays, the dynamic sensorgram obtained by the CCS offers more detailed information on the antibiotic activity at different growth stages ${ }^{37}$. For example, the CCS can show that the presence of ampicillin at a concentration below the MIC will stimulate E. coli growth. Notably, the EAs and mEs of these five antibiotics against E. coli are visibly different from $V$. parahaemolyticus, suggesting that the accuracy of the CCS AST assay also depends on the differences in the testing systems.

Versus automated optical methods, the CCS AST method has a few attractive features. For example, it is unnecessary to remove optical interference substrates from the liquid testing broth because they do not affect the CCS measurements ${ }^{28}$; this leads to a simpler operation. In addition, costs are much lower than optical systems. Electrochemical devices are cheaper than optical instruments because they do not require complex opticalelectrical conversion ${ }^{18}$. The CCS system itself has an approximate price of $\$ 9000$ (USD), which is much cheaper than that of VITEK and Phoenix. By contrast, the cost of assay consumables is rather low. Apart from broths and disposable tubes, neither chemical nor biotic auxiliary materials are needed for the AST assay. The cost of a single assay is no more than $\$ 1$. Moreover, online monitoring to only detect the time point of transition from lag to exponential phase can save many hours ${ }^{9}$. High temporal resolution is important for accurate assays. The temporal resolution of common optical methods is around $10 \mathrm{~min}^{9,38}$. By employing Fourier transform reflectometric interference spectroscopy, the temporal resolution can reach $1 \mathrm{~min}^{37}$. In contrast, CCS collects a conductivity signal every $30 \mathrm{~s}$ to generate growth curves. When required, the temporal resolution can be set higher.

The CCS AST assay requires manual preparation of testing samples and incubation/automated readout. The first step is the same as the BMD AST assay. Operator error is possible because of the requirements for manual processes $^{8}$. This might be the major contribution to the small variation of MICs. However, the preparation of inocula can be easily automated in a commercial version as described ${ }^{15,35}$. In addition, commercial model target antibiotics at a desired amount can be loaded into sample tubes beforehand with mass customization; thus, there are only two fully automated steps in the mature CCS AST assay.

In principle, the proposed CCS AST method successfully addresses issues facing automated optical methods. It does not suffer from interference from complex substrates in the test samples or from adherent cells in proliferation. We believe this enables it wider application fields. In some cases where automated optical methods are inadequate ${ }^{38,42}$ (e.g., blood culture systems, presence of microplastics, nanomaterials and silts in testing system, adherent bacteria), it can work well expectedly.

This study provides proof-of-concept of the phenotypic AST method based on the multichannel CCS. In the presence of antibiotics, dynamic processes of bacterial growth are monitored on-line in a non-destructive manner to generate growth curves. Thus, the MICs of antibiotics against target microorganisms are directly obtained. The total EAs between the CCS AST assays and the reference BMD AST assays are 68.8-92.3\%; this demonstrates the accuracy of the new method for standard bacterial strains and isolates. This approach is superior to the BMD AST method in terms of simplicity, sensitivity and user-friendliness. The sensor itself is affordable. Moreover, the cost for applications is low because it does not involve expensive instruments or auxiliary chemicals. The proposed method provides an automated way to perform AST assays beyond situations where optical methods can be used. It is also a promising high-throughput tool. More validation experiments are planned, e.g., assays in the presence of blood, nanomaterials, silts or micro-plastics. We expect that these experiments will help clinical laboratories develop a versatile platform for rapid MIC determination of diverse types of microorganisms including adherent species.

Received: 14 August 2020; Accepted: 11 November 2020

Published online: 04 December 2020

\section{References}

1. Belkum, A. V. et al. Developmental roadmap for antimicrobial susceptibility testing systems. Nat. Rev. Microbiol. 17, 51-62 (2019).

2. Avesar, J. et al. Rapid phenotypic antimicrobial susceptibility testing using nanoliter arrays. Proc. Natl. Acad. Sci. USA 114, E5787E5795 (2017).

3. Osińska, A. et al. The prevalence and characterization of antibiotic-resistant and virulent Escherichia coli strains in the municipal wastewater system and their environmental fate. Sci. Total Environ. 577, 367-375 (2017).

4. Schumacher, A. et al. In vitro antimicrobial susceptibility testing methods: agar dilution to 3D tissue-engineered models. Eur. J. Clin. Microbiol. Infect. Dis. 37, 187-208 (2018).

5. Boolchandani, M., D'Souza, A. W. \& Dantas, G. Sequencing-based methods and resources to study antimicrobial resistance. Nat. Rev. Genet. 20, 356-370 (2019).

6. Schoepp, N. G. et al. Digital quantification of DNA replication and chromosome segregation enables determination of antimicrobial susceptibility after only 15 minutes of antibiotic exposure. Angew. Chem. Int. Ed. 55, 9557-9561 (2016).

7. Mezger, A. et al. A general method for rapid determination of antibiotic susceptibility and species in bacterial infections. J. Clin. Microbiol. 53, 425-432 (2015).

8. Schoepp, N. G. et al. Rapid pathogen-specific phenotypic antibiotic susceptibility testing using digital LAMP quantification in clinical samples. Sci. Transl. Med. 9, eaal3693 (2017).

9. Veses-Garcia, M. et al. Rapid phenotypic antibiotic susceptibility testing of uropathogens using optical signal analysis on the nanowell slide. Front. Microbiol. 9, 1530 (2018). 
10. Deris, J. B. et al. The innate growth bistability and fitness landscapes of antibiotic-resistant bacteria. Science 342, 1237435 (2013).

11. Syal, K. et al. Current and emerging techniques for antibiotic susceptibility tests. Theranostics 7, 1795-1805 (2017).

12. Zhang, X. et al. Advances in online methods for monitoring microbial growth. Biosens. Bioelectron. 126, 433-447 (2019).

13. Tannert, A. et al. Phenotypic antibiotic susceptibility testing of pathogenic bacteria using photonic readout methods: recent achievements and impact. Appl. Microbiol. Biot. 103, 549-566 (2019).

14. Yang, K. et al. Rapid antibiotic susceptibility testing of pathogenic bacteria using heavy-water-labeled single-cell Raman spectroscopy in clinical samples. Anal. Chem. 91, 6296-6303 (2019).

15. Zhou, M. et al. Comparison of five commonly used automated susceptibility testing methods for accuracy in the China Antimicrobial Resistance Surveillance System (CARSS) hospitals. Infect. Drug. Resist. 11, 1347-1358 (2018).

16. Vourli, S. et al. Evaluation of two automated systems for colistin susceptibility testing of carbapenem-resistant acinetobacter baumannii clinical isolates. J. Antimicrob. Chemother. 72, 2528-2530 (2017).

17. Safavieh, M. et al. Rapid real-time antimicrobial susceptibility testing with electrical sensing on plastic microchips with printed electrodes. ACS Appl. Mater. Interfaces 9, 12832-12840 (2017).

18. Besant, J. D., Sargent, E. H. \& Kelley, S. O. Rapid electrochemical phenotypic profiling of antibiotic-resistant bacteria. Lab. Chip 15, 2799-2807 (2015).

19. Namgyeong, J. et al. Aptamer-functionalized capacitance sensors for real-time monitoring of bacterial growth and antibiotic susceptibility. Biosens. Bioelectron. 102, 164-170 (2018).

20. Ekinci, K., et al. Method and device for antibiotic Susceptibility Testing based on fluctuations of electrical resistance in a microchannel. United States Patent (US 10,214,763 B2) Feb, 2109, 26.

21. Kong, W. et al. Antibacterial evaluation of flavonoid compounds against E. coli by microcalorimetry and chemometrics. Appl. Microbiol. Biot. 99, 6049-6058 (2015).

22. Etayash, H. et al. Microfluidic cantilever detects bacteria and measures their susceptibility to antibiotics in small confined volumes. Nat. Commun. 7, 12947 (2016).

23. Idelevich, E. A. et al. Rapid detection of antibiotic resistance by MALDI-TOF mass spectrometry using a novel direct-on-target microdroplet growth assay. Clin. Microbiol. Infect. 24, 738-743 (2018).

24. Irith, W., Kai, H. \& Robert, E. W. H. Agar and broth dilution methods to determine the minimal inhibitory concentration (MIC) of antimicrobial substances. Nat. Protoc. 3, 163-175 (2008).

25. Jin, W. Y., Jang, S. J. \& Lee, M. J. Evaluation of VITEK 2, microScan, and phoenix for identifcation of clinical isolates and reference strains. Diag. Microbiol. Infect. Dis. 70, 442-447 (2011).

26. Fredborg, M. et al. Real-time optical antimicrobial susceptibility testing. J. Clin. Microbiol. 51, 2047-2053 (2013).

27. Jiang, C. et al. Antifouling strategies for selective in vitro and in vivo sensing. Chem. Rev. https://doi.org/10.1021/acs.chemrev.9b007 $39(2020)$.

28. Zhang, X. et al. Online monitoring of bacterial growth with electrical sensor. Anal. Chem. 90, 6006-6011 (2018).

29. Ahmed, A. et al. Biosensors for whole-cell bacterial detection. Clin. Microbiol. Rev. 27, 631-646 (2014).

30. Settu, K. et al. Impedimetric method for measuring ultra-low E. coli concentrations in human urine. Biosens. Bioelectron. 66, 244-250 (2015).

31. Lin, H. et al. Revisiting with a relative-density calibration approach the determination of growth rates of microorganisms by use of optical density data from liquid cultures. Appl. Environ. Microb. 76, 1683-1685 (2010).

32. Clinical and Laboratory Standards Institute. Methods for dilution antimicrobial susceptibility tests for bacteria that grow aerobically; approved standard-10th ed. CLSI document M07-A10. CLSI Wayne PA, 2015.

33. Kuban, P. \& Hauser, P. C. Fundamental aspects of contactless conductivity detection for capillary electrophoresis. Part I: frequency behavior and cell geometry. Electrophoresis 25, 3387-3397 (2004).

34. Zhang, X. et al. Monitoring acid-base, precipitation, complexation and redox titrations by a capacitively coupled contactless conductivity detector. Measurement 116, 458-463 (2018).

35. Smith, K. P. \& Kirby, J. E. Verification of an automated, digital dispensing platform for at-will broth microdilution-based antimicrobial susceptibility testing. J. Clin. Microbiol. 54, 2288-2293 (2016).

36. Zhang, X. et al. Quantitative determination of target gene with electrical sensor. Sci. Rep.-UK 5, 12539 (2015).

37. Tang, Y. et al. Rapid antibiotic susceptibility testing in a microfluidic pH sensor. Anal. Chem. 85, 2787-2794 (2013).

38. Theophel, K. et al. The importance of growth kinetic analysis in determining bacterial susceptibility against antibiotics and silver nanoparticles. Front. Microbiol. 5, 544 (2014).

39. Patel, J.B., Cockerill, F.R. \& Alder, J. Performance standards for antimicrobial susceptibility testing; twenty-fourth informational supplement. CLSI Wayne PA, 2014.

40. Lopateka, M., Wieczorek, K. \& Osek, J. Prevalence and antimicrobial resistance of vibrio parahaemolyticus isolated from raw shellfish in poland. J. Food Protect. 78, 1029-1033 (2015).

41. Varshney, M. \& Li, Y. B. Double interdigitated array microelectrode-based impedance biosensor for detection of viable Escherichia coli O157:H7 in growth medium. Talanta 74, 518-525 (2008).

42. Zhang, X. et al. Conductometric sensor for viable Escherichia coli and Staphylococcus aureus based on magnetic analyte separation via aptamer. Microchim. Acta 187, 43 (2020).

Acknowledgments

This work was supported by the National Key R\&D Program of China (2019YFD0900505 and 2017YFE1015200).

\section{Author contributions}

X.Z., J.W. and K.Q. designed the study. X.Z., X.J., Q.Y., Y.X., X.W. conducted experiments. X.S. and J.Z. analyzed data. G.X. and Y.Z. helped to isolate bacteria cells from natural samples.

\section{Competing interests}

The authors declare no competing interests.

\section{Additional information}

Supplementary information is available for this paper at https://doi.org/10.1038/s41598-020-77938-7.

Correspondence and requests for materials should be addressed to J.W. or K.Q.

Reprints and permissions information is available at www.nature.com/reprints.

Publisher's note Springer Nature remains neutral with regard to jurisdictional claims in published maps and institutional affiliations. 
(c) (i) Open Access This article is licensed under a Creative Commons Attribution 4.0 International cc) License, which permits use, sharing, adaptation, distribution and reproduction in any medium or format, as long as you give appropriate credit to the original author(s) and the source, provide a link to the Creative Commons licence, and indicate if changes were made. The images or other third party material in this article are included in the article's Creative Commons licence, unless indicated otherwise in a credit line to the material. If material is not included in the article's Creative Commons licence and your intended use is not permitted by statutory regulation or exceeds the permitted use, you will need to obtain permission directly from the copyright holder. To view a copy of this licence, visit http://creativecommons.org/licenses/by/4.0/.

(C) The Author(s) 2020 